\title{
Tunnel Vision: Local Behavioral Influences on Consumer Decisions in Product Search
}

\author{
Gerald Häubl \\ School of Business, University of Alberta, Edmonton, Alberta T6G 2R6, Canada, \\ gerald.haeubl@ualberta.ca \\ Benedict G. C. Dellaert, Bas Donkers \\ Department of Business Economics, Erasmus School of Economics, Erasmus University Rotterdam, \\ 3000 DR Rotterdam, The Netherlands \{dellaert@ese.eur.nl, donkers@ese.eur.nl\}
}

\begin{abstract}
$\mathrm{W}$ e introduce and test a behavioral model of consumer product search that extends a baseline normative model of sequential search by incorporating nonnormative influences that are local in the sense that they reflect consumers' undue sensitivity to recently encountered alternatives. We propose two types of such local behavioral influences that, at each stage of a search process, can manifest themselves both in which of the products inspected up to that point is deemed to be the most preferred one (the product comparison decision) and whether to terminate the search at that stage (the stopping decision). The first of these influences is that consumers respond excessively to the attractiveness of the currently inspected product, at the expense of all others ("focalism"). The second proposed behavioral influence is that consumers overreact to the difference in attractiveness between the current product and the one encountered just prior to it ("local contrast"). Converging evidence from two experiments, which combine to guarantee both high internal and high external validity, provides support for the proposed behavioral influences. Our findings demonstrate that consumers' product comparison and stopping decisions in sequential product search are jointly governed by normative principles and by the proposed local behavioral influences.
\end{abstract}

Key words: search behavior; consumer product search; decision making; behavioral decision theory; consumer behavior

History: Received: June 30, 2008; accepted: June 15, 2009; processed by Robert Meyer. Published online in Articles in Advance September 22, 2009.

\section{Introduction}

Consumer purchase decisions often involve search for a desirable product from a set of relevant products that are available in the market. Analytical work in economics has focused on developing models of optimal search behavior under various circumstances (e.g., Stigler 1961), and some of these normative models have been applied to examine consumer product search (e.g., Putrevu and Ratchford 1997, Ratchford and Srinivasan 1993). Whereas experimental research has shown that normative models of product search represent reasonable first approximations to actual search behavior (Brannon and Gorman 2002; Cox and Oaxaca 1989; Harrison and Morgan 1990; Houser and Winter 2004; Moon and Martin 1990, 1996; Schotter and Braunstein 1981), there is also a body of research that has produced empirical evidence of departures from the decision rules prescribed by such models and that offers competing, behavioral accounts (e.g., Bearden et al. 2005; Hey 1982, 1987; Kogut 1990; Phipps and Meyer 1985; Shu 2008; Sonnemans 1998; Zwick et al. 2003).
Against the background of evidence of both normative and nonnormative determinants of search behavior in different bodies of research, the contribution of the present article is that it presents an integrated behavioral model of search that extends a baseline normative model with specific nonnormative influences. This allows us to examine whether such nonnormative effects should be viewed as complementing the effects prescribed by normative search models, or rather as offering competing alternative explanations of consumer search behavior (e.g., Ben-Akiva et al. 1999). We do so by augmenting a baseline normative model of sequential search by allowing the utilities of products to be systematically affected by nonnormative influences, and at the same time we maintain the basic normative optimization structure of the model. In particular, we incorporate two behavioral influences that reflect different aspects of searchers' undue sensitivity to recently encountered (i.e., "local") alternatives, a type of behavioral effect that is particularly relevant for search where consumers inspect alternatives one at a time (e.g., Hey 1982, Houser and Winter 2004). 
We conceptualize the process of sequential product search as a series of micro decisions, and we model each stage of the search process in terms of two decisions-which of the products inspected up to that stage is deemed to be the most preferred one (the product comparison decision) and whether to terminate the search at that stage (the stopping decision). We suggest that local behavioral influences in sequential search might manifest themselves in both of these micro decisions. In particular, we propose that consumers respond excessively to the attractiveness of the currently inspected product, at the expense of all others ("focalism"), and that they overreact to the difference in attractiveness between the current product and the one encountered just prior to it ("local contrast"). We develop a model of sequential product search that incorporates these proposed behavioral influences in addition to the standard effects implied by the normative theory of search and that also maintains the analytical optimization structure of the normative model.

This behavioral model of consumer product search is tested empirically using detailed process-level data from two Internet-based experiments. Consumer search behavior in Experiment 1 represents a type of search that is common in many real-world settings (e.g., shopping for a stereo system) — sequential search for the best multiattribute product with the possibility of going back to previously inspected products, with limited information about the underlying distribution of utilities, and with the consumer's cost of search manifesting itself in the time and effort spent. Experiment 2 permits a test of our model using evidence gathered in a much more tightly controlled, albeit less realistic, setting. It also involved a sequential search task, but the alternatives were of known utility (i.e., they had a particular monetary value). Participants in Experiment 2 were fully informed about the distribution from which the alternatives' utilities were drawn, and they received a financial reward that was determined by the value of their selected alternative and by the monetary search cost they incurred.

The empirical evidence from these two experiments provides strong support for the proposed behavioral model of consumer sequential product search. Overall, this model is found to provide a significantly better account of the search behavior observed in both experiments than does the baseline normative model. More specifically, the results show that, in making product comparison decisions during sequential search, consumers are unduly influenced by the attractiveness of the currently inspected product, at the expense of all others (focalism effect). In addition, consumers' decisions to either stop or continue searching at a given stage reflect a systematic tendency to overreact to the difference in attractiveness between the current product and the one encountered just prior to it (local contrast effect).

The remainder of the article is organized as follows. First, we develop a baseline normative model of the micro decisions that a consumer makes at each stage of a sequential product search process. Next, we characterize the proposed nonnormative influences and incorporate them into a behavioral model of product search. After that, we report the evidence from two experiments that were designed to test this behavioral model. We conclude with a discussion of our findings and their implications, as well as some suggestions for future research.

\section{Baseline Normative Model of Consumer Product Search}

We examine a common form of consumer product search, where individuals who wish to purchase a particular type of product inspect candidate products that are available in the market one at a time with the aim of identifying the product that best matches their subjective preference (e.g., shopping for a camera or a compact stereo system).$^{1}$ During the search, the utility of a product is fully revealed to the consumer upon its inspection. In line with random utility theory, we assume that consumers evaluate each product in terms of its overall utility to them-i.e., that the various attributes of a product are mapped into a single utility value based on a consumer's multiattribute preference (e.g., McFadden 1986). An important advantage of this approach is that it allows us to tie our model of search in with the rich literature on random utility theory and consumer choice.

The consumer's objective is to maximize her utility taking into account the utility of the purchased product and the total cost of search over all stages of the search process. Every time consumer $i$ inspects a product, she incurs a search $\operatorname{cost} c_{i}$, which is assumed to be constant. ${ }^{2}$ We model the process of consumer product search as a sequence of decision stages $t=$ $1,2, \ldots, T$. At each stage $t$, the consumer makes two decisions: (1) whether the currently inspected product is preferred to the best previously encountered one (the product comparison decision), and (2) whether to terminate the search and purchase the preferred product among those inspected thus far (the stopping decision). Previously observed products can be

\footnotetext{
${ }^{1}$ In contrast to consumer information search (e.g., Erdem et al. 2005, Hauser et al. 1993, Meyer 1982), product search pertains to the case in which the nature of a particular candidate product is unknown to the consumer prior to its inspection.

${ }^{2}$ As is typically the case in real-world consumer search behavior, the cost of search was nonmonetary (i.e., time and effort) in Experiment 1 . By contrast, Experiment 2 used a more controlled setting in which participants incurred a monetary search cost.
} 
"recalled"-i.e., once inspected, a product remains accessible to the consumer as a candidate for purchase for the remainder of the search process.

For the product comparison decision at stage $t$, the consumer compares the utility of the currently observed product $\left(U_{t i}\right)$ to that of the best previously inspected product $\left(U_{t-1, i}^{\max }\right)$. If $U_{t i}$ exceeds $U_{t-1, i}^{\max }$, the new product is recognized as the most attractive one encountered thus far, and $U_{t i}^{\max }$ is updated to take on the value of $U_{t i}$. Otherwise, $U_{t i}^{\max }$ is equal to $U_{t-1, i}^{\max }$. Thus, consumers update the value of the highest product utility they have observed up to that point if

$$
U_{t i}>U_{t-1, i}^{\max } .
$$

Having determined $U_{t i}^{\max }$, consumers decide whether or not to stop their search at the current stage. For this decision, they must know or make assumptions about the distribution of product utilities in the market in order to infer the benefits of continuing the search. Although consumers typically do not know the exact distribution of product utilities in the market, we assume that they (at least) know the utilities of the most and least attractive possible product configurations (i.e., bundles of quality attributes and price) that could be available in the market $\left(U_{\max . i}\right.$ and $U_{\min . i}$, respectively) as they can derive these based on their understanding of the quality attributes and the range of prices, and that this knowledge is not updated over the course of the search. We also assume that consumers ex ante assign equal probabilities to all possible utility values within this range-i.e., that they have a uniform prior-and equal expected utility to all uninspected products. Because the scale of utility is not identified in consumer choice models, we normalize the scale of the range $\left[U_{\min . i}, U_{\max . i}\right]$ to $[0,1]$ for each consumer.

Over the course of the search process, consumers update their beliefs about the distribution of product utilities in the market, $f_{t i}(U)$. In line with prior work pertinent to similar search problems, consumers do so by applying a Bayesian learning rule (e.g., Rothschild 1974). Because the distribution of utilities in the market may not be known to consumers, and for maximum flexibility with respect to distributional shape, we represent this learning process as the Bayesian updating of a Dirichlet process prior, which imposes less stringent assumptions than do approaches based on other commonly used distributions such as the normal (Bikhchandani and Sharma 1996). ${ }^{3}$ Before inspection of the first product, a consumer's belief about the distribution of utilities in the

\footnotetext{
${ }^{3}$ The information that was available to participants in our empirical studies is consistent with this model. However, to examine the robustness of our findings, we also estimated (using the data from Experiment 1) a model that is based on consumers' assumption that
}

market is represented by a prior distribution. The precision of this prior is reflected in its weight $W$, with greater values of $W$ corresponding to less updating of the prior as additional information-in this case, the utility of a newly encountered product-is observed. After each new product inspection, $f_{t i}(U)$ is updated (see the appendix for details).

Under these conditions, the optimal, fully forwardlooking stopping decision at stage $t$ only requires the consumer to determine the expected incremental utility of looking at one additional, previously uninspected product $\left(U_{t i}^{\mathrm{CONT}}\right)$, as has been shown by Rosenfield et al. (1983, Theorem 1). ${ }^{4}$ This is done based on the updated distribution function $f_{t i}(U)$ that accounts for all information about product utilities available at that stage:

$$
U_{t i}^{\mathrm{CONT}}=\int_{U_{t i}^{\max }}^{1}\left(U-U_{t i}^{\max }\right) f_{t i}(U) d U .
$$

Upon termination of the search, the consumer purchases the product with the highest utility among those that she has encountered up to that point $\left(U_{t i}^{\max }\right)$. If the consumer continues the search, she draws an additional, previously uninspected product and then makes a new product comparison decision.

The optimal stopping rule has a reservation utility property (see Rosenfield et al. 1983, Corollary 1). Specifically, the reservation utility $r_{t i}$ is that value of $U_{t i}^{\max }$ that results in a value of $U_{t i}^{\mathrm{CONT}}$ equal to $c_{i}$ at stage $t$, and the consumer stops searching if and only if the highest product utility observed up to that point exceeds the reservation utility. That is, the consumer stops searching if

$$
U_{t i}^{\max }>r_{t i} .
$$

Although the parameters of the Dirichlet process model are updated at each stage of the search, the reservation utilities can be calculated prior to the inspection of the first product (Bikhchandani and Sharma 1996). In fact, as shown in Appendix A, the reservation utility $r_{t i}$ depends only on the consumer's search $\operatorname{cost} c_{i}$, the search stage $t$, and the weight $W$ of the prior, and it can be expressed as

$$
r_{t i}=1-\sqrt{2(W+t) / W} \cdot \sqrt{c_{i}} .
$$

\footnotetext{
the utility of the products is normally distributed with an unknown mean. The estimation results for this alternative baseline normative model are very similar to those for the Dirichlet-based specification, suggesting that our conclusions are not sensitive to the particular distributional assumptions made.

${ }^{4}$ An important property of this structural model is that $U_{t i}^{\mathrm{CONT}}$ is strictly decreasing during the search process, resulting from a nondecreasing $U_{t i}^{\max }$ and a strictly decreasing $f_{t i}(U)$ for $U>U_{t i}^{\max }$. If the cost of search exceeds the expected utility gain from inspecting an additional product at stage $t$, then this also holds for all subsequent stages.
} 


\section{Behavioral Model of Consumer Product Search}

Starting from the normative model presented in the previous section, we propose specific behavioral influences and integrate them into the structural model of sequential product search. Doing so allows us to examine if behavioral effects complement the normative effects or if they offer an alternative explanation for consumer search behavior. The proposed behavioral influences do not affect the normative model's basic optimization structure, thus allowing us to retain this well-established formal aspect of the normative theory of search. This is the case because, given the nature of the underlying cognitive processes, consumers do not anticipate these influences. Within this structure, we include additional, nonnormative components that characterize the proposed behavioral influences on consumers' product comparison and stopping decisions. In the resulting formal behavioral model, the original normative utility terms are replaced by "behavioral" utility terms that capture the proposed departures from the normative model. However, because the normative components of the model are nested within these behavioral terms, the nonnormative extensions can be tested in a relatively straightforward manner. This approach differentiates our model from previous experimental work on nonnormative search behavior, which has focused on developing behavioral models intended to replace, rather than extend, normative models (e.g., Bearden et al. 2005; Hey 1982, 1987; Kogut 1990; Moon and Martin 1990, 1996; Sonnemans 1998; Phipps and Meyer 1985; Zwick et al. 2003).

We focus on behavioral influences that are local in the sense that they reflect consumers' undue sensitivity to recently encountered alternatives, a class of behavioral effects that is particularly relevant for product search where consumers inspect alternatives one at a time. The importance of local influences has been recognized in prior work on search behavior. For instance, Hey $(1982,1987)$ suggests that searchers may apply so-called "bounce rules," which prescribe the initial inspection of a (small) set of alternatives, and the subsequent termination of the search as soon as an additional "draw" reveals an alternative that is more attractive than the best one in the initial set. Phipps and Meyer (1985) propose that stopping errors in search may be due to (satisficing) cutoffs or other suboptimal stopping rules that are local in the sense that they tend not to result in the selection of the overall best alternative. In the context of price search, Houser and Winter (2004) compare a number of different heuristics and find that bounce rules and heuristics that involve stopping after a local winning or losing streak provide a worse fit to the observed search behavior than reservation price heuristics and the normatively optimal search rule. Finally, for sequential search in a setting where only the rank order of the current alternative relative to previously inspected ones can be ascertained, Zwick et al. (2003) report that local properties (i.e., patterns) of the sequence of inspected alternatives can affect how many alternatives individuals inspect before terminating their search.

Against the background of this behavioral work, as well as a separate body of prior research providing evidence of search behavior in line with normative models (Brannon and Gorman 2002, Cox and Oaxaca 1989, Harrison and Morgan 1990, Schotter and Braunstein 1981), the central theme of the present article is that consumers' product search behavior is driven by a combination of normative rules and (predictable) nonnormative influences. This motivates our use of an integrative approach that extends a normative search model with behavioral effects. The key advantage of such an approach is that it allows us to examine the interplay between normative forces and nonnormative influences as they jointly drive product comparison decisions as well as stopping decisions in consumer search.

\section{Local Behavioral Influences in Sequential Search} We propose that the particular nature of sequential product search gives rise to specific types of behavioral influences on consumers' decisions in this domain. A key property of sequential search is that alternatives are encountered one at a time, with consumers having to make a product comparison decision and a stopping decision at each search stage. Therefore, the relative order position of products in the sequence is likely to be a key behavioral driver of how specific products are evaluated. In particular, we propose that consumer decision making in sequential product search is susceptible to behavioral influences that are local in the sense that they reflect undue sensitivity to recently encountered alternatives. We examine two types of such local influences-focalism and local contrast-that might manifest themselves both in product comparison decisions and in stopping decisions. ${ }^{5}$ We discuss each of these influences in turn.

Focalism. Research in psychology has shown that people tend to overweight whatever information is most salient or most accessible at a particular moment and neglect other relevant considerations (see, e.g., Kahneman and Miller 1986, Schwarz 1990, Wilson

\footnotetext{
${ }^{5}$ Whereas other types of local influences are possible, we focus on these two because they are the most elementary ones in the sense that more complex effects could be constructed using these as basic building blocks.
} 
et al. 2000). Such an inclination to be unduly influenced by a focal stimulus or event has been demonstrated in a variety of domains, including judgments of subjective well-being and happiness (Schkade and Kahneman 1998, Suh et al. 1996), predictions about one's own future experiences (Gilbert and Wilson 2007, Wilson and Gilbert 2005), and the estimation of task completion times (Buehler et al. 1994, Kruger and Evans 2004).

The tendency to be unduly influenced by focal information might also manifest itself in sequential product search. In particular, we propose that at a given search stage, consumers place greater weight on (the attractiveness of) the currently inspected product than they ought to normatively when making their product comparison and stopping decisions. This prediction is also consistent with research on order effects suggesting that decision makers tend to attach greater weight to more recently encountered information when they-as in sequential product search-obtain and evaluate information in a step-by-step fashion (Hogarth and Einhorn 1992, Johar et al. 1997).

With respect to product comparison, according to the baseline normative model, the consumer's decision should be driven by the difference in utility between the currently inspected product and the best previously encountered one, with equal weight given to both of these. Even though we expect this utility difference to have the effect implied by the normative model, we propose that, in addition, the product comparison decision is influenced more strongly by the utility of the current product than by that of the best product encountered prior to that.

With respect to stopping, the normative model prescribes that the consumer's decision should be affected by the attractiveness of the currently inspected product only if the latter is the most attractive one observed thus far. Focalism in this decision suggests a difference in the impact of the utility of the product that is currently inspected compared to the utility of a product that is retrieved from memory. As the stopping decision is driven by the utility of the best product observed thus far, focalism will only affect this decision if the currently inspected product is also the best one observed up to that point. In particular, we propose that the utility of the best product thus far will have a larger influence on the stopping decision if it is the currently inspected one than when the best product was observed earlier in the search process and, thus, has to be recalled from memory.

Local Contrast. Work in cognitive psychology has revealed that, when evaluating alternatives in sequence, individuals unintentionally use recently encountered alternatives as reference points along with more stable background reference points (Heyman et al. 2004, Mellers 2000). This is also consistent with the classic finding in the area of visual perception that judgments of brightness reveal a local contrast effect such that a stimulus appears darker when it is adjacent to a brighter stimulus, and vice versa (Arend and Spehar 1993, Pessoa et al. 1995, Reid and Shapley 1988). In line with this prior work, we propose a local contrast effect whereby consumers' perception of a newly encountered product's attractiveness in sequential search is influenced by how that product compares to the one inspected just prior to it.

We propose that consumer decision making in sequential product search is influenced by the local contrast (in terms of overall attractiveness) between the currently inspected product and the one encountered just prior to it, even when the latter is normatively irrelevant. We propose that such a local contrast effect might manifest itself both in the product comparison decision and in the stopping decision.

For product comparison, the baseline normative model prescribes that the consumer's decision at a given search stage should only be influenced by the utility of the currently inspected product and that of the best among all previously encountered products. Thus, the product seen just prior to the current one should not affect this decision, unless it happens to have been the most attractive one encountered up to that point. However, we predict that the local contrast between the current product and the one inspected before it influences the product comparison decision even when, normatively, it should have no such effect.

With respect to stopping, according to the normative model, the consumer's decision at a given stage should be driven only by the utility of the most attractive product encountered up to that point and the reservation utility (see Equation (3) above). By contrast, we predict that the stopping decision is also influenced by the difference in utility between the current product and the one inspected just prior to it-even when the latter is normatively irrelevant. In particular, in case of a positive local contrast, this enhances the utility of the best product encountered thus far, making it more likely that this utility exceeds the reservation utility. Consequently, a local improvement renders consumers more likely to stop at the current stage of the search. On the other hand, we do not expect a negative local contrast to affect the stopping decision because this decision is driven only by the evaluation of the best product observed thus far.

\section{Micro Decision Models with Behavioral Influences}

We incorporate the proposed behavioral influences into the structural model as follows. As consumers do not anticipate these influences, the normative model's optimization structure is maintained (i.e., the reservation utility property holds). The behavioral influences can thus be directly integrated into 
the structural model by using behavioral counterparts of the currently inspected product's utility in the decision rule stated in Equation (1) and of the best product's utility in the decision rule expressed in Equation (3). More specifically, to capture the focalism and local contrast effects, we allow the utility of a given product to be a function of these behavioral influences. We refer to this as the "behavioral utility" $(B U)$ of a product, which replaces the normative utility in the structural model. Because the product comparison and stopping decisions differ in their focus, the behavioral effects are not assumed to be equal across them.

The behavioral influences are incorporated into the product comparison model as follows. The behavioral utility of the currently inspected product $\left({ }^{P C} B U_{t i}\right)$ is expressed as a function of both $U_{t i}$ and two behavioral components. To capture the proposed focalism effect, an additional weight is placed on the utility of the most attractive product observed thus far if it is the current one $\left(U F O C A L_{t i}\right)$, reflecting a greater sensitivity to the attractiveness of the currently inspected product relative to that of previously encountered products. In addition, to capture the proposed local contrast effect, ${ }^{P C} B U_{t i}$ is allowed to be influenced by the difference in utility between the current product and the one inspected just prior to it, i.e., $\left|U_{t i}-U_{t-1, i}\right|$. We allow for asymmetry in this local contrast effect by distinguishing between the case in which the current product is better $\left(\operatorname{LOCPOS}_{t i}\right)$ and that in which it is worse $\left(L O C N E G_{t i}\right)$ than the previous one. In the product comparison decision, the behavioral utility of the current product $\left({ }^{P C} B U_{t i}\right)$ is compared to the utility of the best previously inspected product $\left(U_{t-1, i}^{\max }\right)$ to determine whether or not the latter should be updated (see Equation (1)): ${ }^{6}$

$$
\begin{gathered}
{ }^{P C} B U_{t i}>U_{t-1, i}^{\max }, \\
{ }^{P C} B U_{t i}=U_{t i}+\gamma_{F}^{P C} U_{F O C A L_{t i}}+\gamma_{P}^{P C} \text { LOCPOS }_{t i} \\
+\gamma_{N}^{P C} \text { LOCNEG }_{t i} .
\end{gathered}
$$

In the normative stopping model, the utility of the most attractive product observed thus far drives the consumer's decision to either stop or continue the search. The behavioral equivalent of this utility $\left({ }^{S} B U_{t i}^{\max }\right)$ is expressed as a function of both $U_{t i}^{\max }$ and the two behavioral influences. Note that in the stopping model, focalism only enters into the model if the current product is the most attractive one observed thus far. If this is not the case, the utility of the current product is irrelevant to the stopping decision because $U_{t i}^{\max }$ is not updated and the stopping decision is based on the utility of the best previously

${ }^{6}$ The utility of the best product observed up to stage $t-1$ is assumed to be unaffected by local behavioral influences at stage $t$. inspected product. To capture the proposed focalism effect, an additional weight is placed on the utility of the most attractive product observed thus far if it is the current one $\left(U F O C A L_{t i}\right)$. For local contrast, $L O C N E G_{t i}$ is not included because a local deterioration logically precludes the possibility of the current product being the best one encountered thus far. Consequently, $\operatorname{LOCPOS}_{t i}$ is the only variable used to capture a local contrast effect in the stopping model. The (updated) behavioral utility of the best product observed thus far $\left({ }^{S} B U_{t i}^{\max }\right)$ is compared to the reservation utility $\left(r_{t i}\right)$ in making the stopping decision (see Equation (3)):

$$
\begin{gathered}
{ }^{s} B U_{t i}^{\max }>r_{t i}, \\
{ }^{s} B U_{t i}^{\max }=U_{t i}^{\max }+\gamma_{F}^{S} U F O C A L_{t i}+\gamma_{P}^{S} \text { LOCPOS }_{t i} .
\end{gathered}
$$

To summarize, we incorporate the local behavioral influences into the structural model by adding them to the normative utility of the currently inspected product in the product comparison model and to that of the best product encountered up to that point in the stopping model.

\section{Experiment 1: Test of the Model in a Naturalistic Setting}

As a first test of the proposed behavioral model of consumer product search, we conducted an experiment involving a sequential product search task that resembled a typical prepurchase search activity and that concluded with the choice of one of the available products or services.

\section{Method}

Sample. Participants were recruited from a consumer panel maintained by a major university in The Netherlands. The members of this panel had been selected randomly from the national population of The Netherlands. Participants completed the study from their home, using personal computers connected to the Internet.

One week prior to the actual experiment, prospective participants completed a brief Internet-based screening questionnaire. This involved measures of their degree of interest in two product categoriescompact stereo systems and holiday home rental packages. Based on this screening study, members of the consumer panel who had expressed an interest in at least one of the categories, and who were over the age of 16 , were selected for potential participation in the actual study and scheduled to complete the latter in connection with their category of greatest interest.

In the main study, usable responses were obtained from 115 consumers. The numbers of participants who completed the experiment in connection with 
compact stereo systems and holiday home rental packages were 48 and 67 , respectively. The average age of participants in the study was 46.8 years, $41.7 \%$ were female, and $47.8 \%$ held a bachelor's (or higher) academic degree.

Procedure. At the start of the experiment, participants were automatically assigned to the product category of greatest interest to them based on their responses in the screening study. They first read detailed instructions describing the attribute dimensions used to characterize the products in the assigned category and explaining, among other things, the nature of the tasks they were about to perform. After that, participants completed a task that was designed to measure their multiattribute preference in the focal product category (see below for a detailed description of this task). They then proceeded to the productsearch-and-choice task.

The search environment closely resembled an Internet site listing different products available from an online retailer. This interface allowed participants to examine stereo systems or holiday home packages that were available in a hypothetical market (see the next section for details). The alternatives were listed in arbitrary order, randomized independently for each participant. The list, which contained uninformative (randomly generated) product ID numbers, was partitioned into screens of 25 products each. Participants were told that the products were listed in random order and that their goal was to indicate which one of these they would choose. Apart from participants' time and effort, there were no costs associated with searching. Participants were able to request the detailed description - in terms of six quality attributes and price - of any of the products by a mouse click. Thus, they were free to search the list in any order. All alternatives remained available throughout the search, but a detailed description could be displayed for only one product at a time. Participants were encouraged (but not forced) to use a feature of the interface that allowed them to mark their "current favorite"-i.e., the subjectively most attractive product they had found up to that point-at each stage of the search. ${ }^{7}$

Once participants had decided to terminate their search and make their product choice, they clicked on

\footnotetext{
${ }^{7}$ For the purpose of model estimation, it was assumed that the first inspected product was the most attractive one until another product was marked as the current favorite. Of the 115 participants, 86 used this interface feature at least once. Of the 29 who did not, 24 searched fewer than seven products, which may explain why they did not use the feature. We also estimated our proposed model separately for only those individuals who had used the interface feature for indicating the current favorite and found that the results were very similar to those obtained for the full sample, which are reported in the article.
}

a link identifying their preferred product. They then saw a choice confirmation screen, which contained the detailed description of that product. Finally, they were debriefed and thanked for completing the study.

Hypothetical Product Market. Participants performed the product-search-and-choice task in a hypothetical market consisting of 500 products, each described in terms of six quality attributes and price. The quality attributes were varied at either two or five levels, and each attribute had the property that its levels could be rank ordered in terms of their objective attractiveness. ${ }^{8}$ The quality attributes used to describe holiday home rental packages were privacy level, design and landscaping of the park, and sauna availability (all at two levels), as well as independent quality rating, swimming pool size, and sports facilities (all at five levels). The corresponding attributes for compact stereo systems were number of CD slots, number of cassette decks, and quality of the speaker system (all at two levels), as well as power of the speaker system, number of programmable channels, and independent sound quality rating (all at five levels).

The product market was constructed as a half fraction of a $2^{3} 5^{3}$ full-factorial design for the six quality attributes. Each of the resulting product profiles was subsequently assigned a price based on additive component prices for its attribute levels. This produced a strong positive correlation between price and quality, which is typical of real-world markets and ensured that none of the products was objectively dominated by any other product. Finally, a small random component was added to each product's price (resulting in a random perturbation of product prices of about $1 \%$ on average), which guaranteed that there was a nondeterministic relationship between price and quality.

Utility of Inspected Products. Calculation of the utility to consumer $i$ of an inspected product (at stage $t$ of the search process), $U_{t i}$, requires an understanding of that person's multiattribute preference. Prior to the product-search-and-choice task, participants completed a ratings-based conjoint task that was designed to measure their preference in the focal product category. Descriptions of eight products had been constructed according to a fractional orthogonal design involving six quality attributes and price, all manipulated at two levels-the most and the least attractive level that would subsequently be available in the market. During the preference measurement task, participants rated each of these eight products,

\footnotetext{
${ }^{8}$ For all participants, the a priori rank orders of the levels of all attributes were confirmed by the individual-level part-worth utilities that were estimated based on their responses during the preference measurement task.
} 
which were presented one at a time, on an 11-point scale $(0=$ "very unattractive" to $10=$ "very attractive"). This allowed the estimation of individuallevel part-worth utilities for each of the six quality attributes and for price based on a participant's ratings of the eight product profiles (e.g., Elrod et al. 1992). ${ }^{9}$ For each individual, these part-worth utilities were then used, in conjunction with a product's attribute levels and price, to calculate an approximate utility score (based on a linear weighted additive utility model) for each of the products. The range of utility scores, from the least to the most attractive possible product configuration, was normalized to $[0,1]$ for each participant.

Dependent Variables. The dependent variables in the stopping and product comparison models are operationalized as follows. First, at each stage of the search process, we observed whether or not a participant marked the newly inspected product as his or her most preferred one among those inspected up to that point, which is measured by Prefer $_{t i}$. More specifically, each stage of the search process provided a binary observation of which product was the most preferred one to the participant at that stagethe newly inspected product or the best previously encountered one. Second, at each stage of the search process, we also observed whether or not a participant chose to stop searching, captured in Stop $t_{t i}$ having the value of one and zero, respectively.

Model Specification. The fully deterministic version of participants' product comparison and stopping decisions is unlikely to perfectly describe behavior. Its estimation requires a richer, stochastic specification. To allow for systematic differences across individuals, and hence account for the fact that we have multiple observations for each participant, we include two individual-specific random effects in the model. The first, $v_{i}^{P C}$, enters in the product comparison equation to allow for heterogeneity in individuals' tendency to mark the current product as the best one observed thus far. The second, $v_{i}^{S}$, enters in the search cost equation and captures systematic differences in search costs across individuals. In addition, $v_{i}=\left(v_{i}^{P C}, v_{i}^{S}\right)$ is assumed to follow a mean-zero bivariate normal distribution with arbitrary variance-covariance matrix,

\footnotetext{
${ }^{9}$ To examine the robustness of our findings, we also used an empirical Bayes approach (see, e.g., Morris 1983) as an alternative method of obtaining the individual-level part-worth utilities. The population distribution of the part-worth utilities, estimated via a random coefficient model, served as the prior distribution for each individual and was updated using the individual's ratings to obtain individual-level part-worth utilities. Using these alternative partworth utilities in calculating the product utilities did not alter our conclusions regarding the proposed behavioral influences.
}

hence allowing for correlation between its components. Any remaining unexplained variation is captured by independent and identical extreme value type I error terms, $\varepsilon_{t i}^{P C}$ and $\varepsilon_{t i}^{S}$, resulting in a Logittype model.

The empirical model for the product comparison decision, combining Equations (1) and (5) and including $v_{i}^{P C}$ and $\varepsilon_{t i}^{P C}$, is

$$
\begin{aligned}
V_{t i}^{P C}= & U_{t i}+\gamma_{F}^{P C} \text { UFOCA }_{t i}+\gamma_{P}^{P C} \text { LOCPOS }_{t i} \\
& +\gamma_{N}^{P C} \text { LOCNEG }_{t i}-U_{t-1, i}^{\max }+\nu_{i}^{P C}, \\
\text { Prefer }_{t i} & =I\left(V_{t i}^{P C}+\varepsilon_{t i}^{P C}>0\right) .
\end{aligned}
$$

In the stopping model, an individual's cost of search plays an important role because the reservation utility equals $1-\sqrt{2(W+t) / W} \cdot \sqrt{c_{i}}$ (see Equation (4)). ${ }^{10}$ We capture a participant's systematic search cost $c_{i}$ by both the (unobserved) mean level of search cost among all participants $\left(\gamma_{\text {search }}\right)$ and an observed proxy for that individual's opportunity cost of time (TIMECOST $)_{i}$ :

$$
\sqrt{c_{i}}=\gamma_{\text {search }}+\gamma_{\text {time }} \text { TIMECOST }_{i}+\nu_{i}^{S} .
$$

Combining Equations (4), (6), and (8) and incorporating $v_{i}^{S}$ and $\varepsilon_{t i}^{S}$, yields the following specification of the stopping model:

$$
\begin{aligned}
& V_{t i}^{S}=U_{t i}^{\max }+\gamma_{F}^{S} \text { UFOCA }_{t i}+\gamma_{P}^{S} \text { LOCPOS }_{t i} \\
&-(1-\sqrt{2(W+t) / W} \\
&\left.\cdot\left(\gamma_{\text {search }}+\gamma_{\text {time }} \text { TIMECOST }_{i}+\nu_{i}^{S}\right)\right), \\
& \text { Stop }_{t i}= I\left(V_{t i}^{S}+\varepsilon_{t i}^{S}>0\right) .
\end{aligned}
$$

To allow for the usual variance restriction on epsilon in choice models, we rescale the model. We do so by estimating a set of $\beta$ coefficients that comprises scale corrections of the $\gamma$ coefficients and of the previously unparameterized model components. This results in the following two equations:

$$
\begin{aligned}
V_{t i}^{P C}= & \beta^{P C}\left(U_{t i}-U_{t-1, i}^{\max }\right)+\beta_{F}^{P C} U_{F O C A L_{t i}} \\
& +\beta_{P}^{P C} \text { LOCPOS }_{t i}+\beta_{N}^{P C} \text { LOCNEG }_{t i}+\nu_{i}^{P C}, \\
V_{t i}^{S}= & \beta^{S}\left(U_{t i}^{\max }-1\right)+\beta_{F}^{S} U_{F O C A L_{t i}}+\beta_{P}^{S} \text { LOCPOS }_{t i} \\
& +\sqrt{2(W+t) / W} \cdot\left(\beta_{\text {search }}+\beta_{\text {time }} \text { TIMECOST }_{i}+\nu_{i}^{S}\right) .
\end{aligned}
$$

The total likelihood of the joint decision models is

$$
\begin{aligned}
& \prod_{i} \int \prod_{t=1}^{T_{i}}\left[P\left(V_{t i}^{P C}+\varepsilon_{t i}^{P C}>0\right)^{\text {Prefer }_{\text {ti }}}\right. \\
& \cdot\left(1-P\left(V_{t i}^{P C}+\varepsilon_{t i}^{P C}>0\right)\right)^{1-\text { Prefer }_{t i}} \cdot P\left(V_{t i}^{S}+\varepsilon_{t i}^{S}>0\right)^{\text {Stop }_{t i}} \\
& \left.\quad \cdot\left(1-P\left(V_{t i}^{S}+\varepsilon_{t i}^{S}>0\right)\right)^{1-\text { Stop }_{t i}}\right] \varphi\left(v_{i}\right) d v_{i} .
\end{aligned}
$$

\footnotetext{
${ }^{10}$ In estimation, the weight $W$ of the prior was set to an arbitrary value (10). Estimation of our model for different levels of $W$ does not change the substantive conclusions.
} 
To estimate the model including the unobserved heterogeneity across individuals embedded in $v_{i}$, we rely on the method of simulated maximum likelihood (Train 2003). This involves the sampling of a large number of draws (in our case 100) from the distribution of $v_{i}$ (which depends in part on parameters that are also estimated) and the computation of the likelihood conditional on these draws. The likelihood of Equation (11) is then approximated by averaging the computed likelihood conditional on each of the draws across all the draws. Note that these draws differ across individuals and that the likelihood conditional on $v_{i}$ is smooth, thus improving the properties of the estimator (Stern 1997).

Predictor Variables in the Product Comparison Model. Intercept: The intercept in the product comparison model represents the mean baseline level of individuals' tendency to select the current product (i.e., the one inspected at stage $t$ ) as the most attractive one encountered thus far.

Utility difference between current and best previously inspected product: This utility difference variable, which should drive the product comparison decision according to the normative model, is based on the preference measurement procedure described above, and operationalized as the difference between the utility of the current product and that of the best previously encountered one $\left(U_{t i}-U_{t-1, i}^{\max }\right)$.

Focalism: The predicted overweighting of the currently inspected (i.e., focal) product is tested by allowing for a differential impact of the utility of that product relative to the utility of the best previously encountered product. To that end, we include the predictor variable $U F O C A L_{t i}$ that is equal to the utility of the current product. An effect of UFOCAL $L_{t i}$ indicates a behavioral influence of the current product over and above the normative influence of the utility difference between the current and the best previously inspected product.

Local contrast: The proposed local contrast effect is captured in terms of the difference in utility between the current product and the one inspected just prior to it $\left(U_{t i}-U_{t-1, i}\right)$. In the product comparison model, we allow for asymmetry in this effect depending on whether the difference is positive (i.e., a local "improvement") or negative (i.e., a local "deterioration"), and we do so by including the predictor variables LOCPOS $t i$ and LOCNEG respectively.

Predictor Variables in the Stopping Model. The two normative drivers of the decision to terminate the search at a given stage are the utility of the best product encountered up to that point, $U_{t i}^{\max }$, and the reservation utility, $r_{t i}$. A consumer's reservation utility is a function of the stage in the search process, the weight $W$ of his or her prior belief about the distribution of utilities in the market, and his or her cost of search. The latter is captured in the model through a common (i.e., not individual-specific) baseline search cost and a time-based measure of an individual's cost of search. The search stage $t$ and the prior weight $W$ enter the model through the searcher's updated belief about the distribution of utilities, and consequently, they do not appear as explanatory variables in the estimated model (see Equation (8)).

Intercept: The intercept in the stopping model is identified separately from the baseline search cost, as the impact of the cost of search decreases over the course of the search process by a factor $(W+t) / W$.

Correction for utility normalization: The preference measurement task provides us with a cardinal value for the utility of each product, and the product utilities are normalized to be between zero and one. This normalization is arbitrary as both the scale and location of the utilities are not identified. Any alternative scaling of the utilities results in a corresponding rescaling of the estimated parameters, with no need for further correction. A shift in the location of the utilities, however, does require an explicit correction. To absorb the impact of such a shift in the utility location embedded in this variable, we include a dummy variable that takes on a value of one if the focalism variable is nonzero. ${ }^{11}$ (Note that such a correction is not necessary in the product comparison model.)

Baseline search cost: This is the intercept in the search cost equation part of the stopping model. It captures the mean baseline level of the cost of inspecting one product across individuals.

Time-based search cost: A participant's average response time (across the eight products) during the preference measurement task that preceded the search task is used as an (inverse) indicator of that individual's opportunity cost of time. This indicator increases the individual's search cost beyond the baseline level.

Utility of best inspected product: The utility of the most attractive product among all those examined up to, and including, the current stage $\left(U_{t i}^{\max }\right)$.

Focalism: The predictor variable UFOCAL $L_{t i}$, operationalized as in the product comparison model, is also used to capture the predicted focalism effect on the stopping decision. Note, however, that the currently inspected product only enters the stopping model if it is the best product encountered up to that point.

Local contrast: In the stopping model, $L_{O C P O S}$ is the only variable used to capture a local contrast effect (see Equation (6)).

\footnotetext{
${ }^{11}$ This dummy variable is identical to the Prefer variable as it takes on a value of 1 whenever the currently inspected product is selected as the best one encountered thus far.
} 


\section{Results}

Descriptive Statistics. On average, participants inspected the descriptions of 14.3 different products during the product-search-and-choice task. The average time they spent looking at these descriptions was 45.8 seconds per product. The average utility (on a scale from zero to one, normalized for each participant over all available products) of the best product that participants encountered in their search was 0.783 , whereas that of the product they chose was 0.665 . There were no significant differences on these measures between individuals who completed the task for compact stereo systems and those who did so in connection with holiday home rental packages.

Product Comparison Decision. The results for the product comparison model are summarized in the top portion of Table 1. First, the normative theory of search suggests that a consumer's decision as to whether the currently inspected product is more attractive to him or her than the best previously encountered one should be based only on the difference in utility between these two alternatives. This is supported by our data $(\beta=2.72, t=4.89)$. The greater the utility difference in favor of the current productor the smaller the difference in favor of the best prior one-the more likely a consumer is to select the current alternative either for immediate purchase (if the search is terminated at the current stage) or as the most preferred one up to that point (if the search continues).

The first proposed behavioral influence on the product comparison decision in sequential search suggests that consumers are unduly influenced by the attractiveness of the currently inspected product. The

\section{Table 1 Model Estimates (Experiment 1)}

\begin{tabular}{|c|c|c|c|}
\hline \multicolumn{2}{|c|}{ Predictor variable } & $\beta$ & $t$-value \\
\hline \multicolumn{4}{|c|}{ Product comparison model } \\
\hline & Intercept & $-2.88^{*}$ & -5.55 \\
\hline N & $\begin{array}{l}\text { Utility difference between current and } \\
\text { best previously inspected product }\end{array}$ & $2.72^{*}$ & 4.89 \\
\hline B & Focalism (differential impact of current product) & $2.96^{*}$ & 3.83 \\
\hline B & Local contrast: Improvement vs. prior product & 0.11 & 0.25 \\
\hline \multirow[t]{2}{*}{ B } & Local contrast: Deterioration vs. prior product & 1.28 & 1.04 \\
\hline & $\sigma\left(v^{P C}\right)$ & $0.66^{*}$ & 4.28 \\
\hline \multicolumn{4}{|c|}{ Stopping model } \\
\hline & Intercept & $9.86^{*}$ & 3.98 \\
\hline & Correction for utility normalization & $-4.32^{*}$ & -2.36 \\
\hline & Baseline search cost & $4.49^{*}$ & 3.22 \\
\hline $\mathrm{N}$ & Time-based search cost & $-0.19 *$ & -2.17 \\
\hline N & Utility of best inspected product & 1.94 & 1.66 \\
\hline & Focalism (differential impact of current product) & 4.74 & 1.88 \\
\hline \multirow[t]{2}{*}{ B } & Local contrast: Improvement vs. prior product & $2.07^{*}$ & 3.10 \\
\hline & $\sigma\left(v^{S}\right)$ & $0.16^{*}$ & 2.00 \\
\hline
\end{tabular}

Note. N: normative influence; B: behavioral influence.

*Parameter estimate significant at $p<0.05$. empirical evidence obtained in Experiment 1 provides support for such a focalism effect in product comparison. In contrasting the utility of the current product with that of the best previously inspected one, a change in the current product's utility has a stronger impact $(\beta=2.96, t=3.83)$ on the probability of selecting the current product as the best one thus far than an equivalent change (in the opposite direction) in the utility of the best previously encountered one.

The second candidate behavioral influence is that of the difference in utility between the currently inspected product and the one seen just before that, when the latter is not the best among the previously observed products and is, thus, normatively irrelevant. Such local contrasts are not significant in the product comparison model, both when they represent an improvement and when they constitute a deterioration relative to the prior product.

Stopping Decision. The results for the stopping model are also summarized in Table 1 . According to the normative theory of search, the stopping decision should be based only on the cost of search and on the utility of the most attractive product examined thus far. The effect of the latter is in the expected direction, approaching statistical significance (and it is significant at $p<0.05$ for a one-tailed test). Moreover, the time-based measure that captures differences in search cost across individuals has a significant effect in the direction predicted by the normative theory-those who spent more time on the preference measurement task that preceded the search task (as indicated by the variable Time-based search cost) were less likely to terminate the search at a given stage $(\beta=-0.19, t=-2.17)$.

The first of the two proposed behavioral influences on the stopping decision is that consumers overweight the attractiveness of the current product. This implies the prediction that the utility of the best among all products encountered thus far has a stronger (positive) effect on the probability of terminating the search at a given stage when that product happens to be the currently inspected one (which is captured by the current product's behavioral utility, ${ }^{S} B U_{t i}$, in our model). This focalism effect $(\beta=4.74$, $t=1.88$ ) approaches statistical significance (and it is indeed significant at $p<0.05$ for a one-tailed test, reflecting our directional hypothesis for it).

Moreover, we have proposed that the probability of stopping the search at a given stage is positively influenced by the local improvement in utility in this product relative to the one encountered immediately prior to it, which is normatively irrelevant. This local contrast effect is significant in the stopping model $(\beta=2.07, t=3.10)$, suggesting that, all else being equal, observing a product that is superior to the one they saw just before it renders consumers more 
likely to terminate their search. Note that our specification of the normative part of the stopping model based on a Dirichlet process results in a conservative test of the nonnormative nature of the local contrast effect. In particular, a plausible alternative account of normative stopping behavior-based on consumers assuming a normal distribution of utilities and learning about its variance-would imply that observing a highly unattractive product in a search sequence may increase the probability of subsequently encountering a highly attractive one. In that case, greater variance among a set of inspected products should result in a lower probability of stopping-counter to the local contrast effect, which predicts that greater local differences in utility increase the probability of stopping at a given search stage.

Comparative Model Fit. As an overall test of the behavioral influences, we also estimated the baseline normative model as a benchmark. This model was identical in structure to the full behavioral model but differed from the latter in that none of the behavioral effects were included. Based on a likelihood-ratio test, the behavioral model fits the data significantly better than the baseline normative model $\left(\chi^{2}=47.2, d f=6\right.$, $p<0.001)$. A comparison of these two models in terms of the Bayesian Information Criterion (BIC)1845.3 for the behavioral model versus 1864.1 for the normative model-yields the same conclusion. Thus, the local behavioral influences, taken together, contribute substantially to explaining the observed product comparison and stopping decisions. Moreover, to test for heterogeneity in the behavioral effects across individuals, we also estimated a latent-class model with class-specific coefficients for both the normative and the behavioral influences. For these data, a model with two latent classes is inferior (BIC of 1869.1) to the homogeneous behavioral model (BIC of 1845.3). Finally, as an absolute measure of fit, McFadden's $R$-squared for the behavioral model is 0.12 .

\section{Discussion}

The results of Experiment 1 provide support for the proposed behavioral model and, thus, suggest that consumers are indeed susceptible to nonnormative local influences during product search. Consumers' undue sensitivity to recently encountered alternatives is reflected both in the product comparison decisions and in the stopping decisions they make at each search stage. In particular, product comparison decisions reflect strong focalism effects, whereas stopping decisions are largely driven by local contrast effects.

To illustrate the magnitude of the key behavioral influences observed in Experiment 1, we examine how variation in the variables that capture these influences affects consumers' micro decisions in sequential product search. We do so based on the estimated parameters of the product comparison and stopping models reported in Table 1. For a given behavioral effect, we calculate the probability of selecting the currently inspected product as the most preferred one thus far or the probability of stopping the search at the current stage for different percentiles of the observed distribution of the variable characterizing the effect. Each behavioral effect is examined ceteris paribus, with all other variables in the model held constant.

First, to illustrate the focalism effect on product comparison decisions, we examine the probability of the current product being adopted as the most preferred one thus far for both the 10th and the 90th percentile values of this product's utility when the latter is equal to the utility of the best previously encountered product. Normatively, when the current and the best prior product are equally attractive, consumers' inclination to prefer the current product should not be affected by the absolute attractiveness of these two products. However, the resulting probabilities are in fact substantially different-they are $11.6 \%$ and $37.5 \%$ for the 10th and the 90th percentiles, respectively. Thus, our findings reveal a greater sensitivity to the utility of the current product than to that of the best prior one. To further quantify this behavioral influence, our model estimates imply that if the best previously encountered product was at the median of the utility distribution, the currently inspected one would only have to be at the 26th percentile to be equally likely to be selected as the most preferred product thus far.

A similar approach can be used to illustrate the local contrast effect on stopping decisions. We examine the probability of terminating the search at the current stage for both the 10th and the 90th percentile values of the utility of the product inspected just prior to the current one while holding both the utility of the current product and that of the best product encountered thus far constant at the 90th percentile. The resulting stopping probabilities are $66.0 \%$ and $49.9 \%$ for the 10th and the 90th percentile values of the previous product's utility, respectively. This provides a sense of the magnitude of the local contrast effect because, normatively, the attractiveness of the product encountered just prior to the current one should not affect a consumer's inclination to terminate the search. To further quantify this effect, if the previous product was at the 10th percentile of the utility distribution, the current product would only have to be at the 35th percentile to achieve the same stopping probability as when the utilities of both the current and the previous product are at the median. Thus, all else being equal, a local increase in attractiveness from one inspected product to the next unduly renders consumers more likely to terminate their search. 
Finally, it is worth noting that the normative effects are also significant both in the product comparison model and in the stopping model, which suggests that the proposed local behavioral influences augmentrather than replace-the normative theory of sequential search.

\section{Experiment 2: Test of the Model in a Controlled Setting}

Experiment 1 used an experimental paradigm that was quite naturalistic - consumers searched a set of multiattribute alternatives in a domain of interest to them and concluded their search with the choice of a product according to their own, idiosyncratic preferences. Whereas such an approach has a number of desirable features (including high external validity), it also has some limitations. In particular, it does not allow the researcher to directly observe consumer search costs and returns to search, and it requires the use of a highly flexible learning model to characterize consumers' assumptions about the distribution of utilities in the market at each stage of the search. The purpose of Experiment 2 was to test the proposed behavioral model of sequential search in a much more controlled setting. To that end, we used alternatives of unambiguous utility (i.e., monetary value), with the latter being drawn from a distribution known to participants. Moreover, searchers were charged a monetary cost for inspecting each new alternative, and they received a financial reward based on the value of the alternative they chose and the total search cost they incurred. This paradigm allowed us to define a straightforward normative model, calculate exact reservation values, and determine whether the observed product comparison and stopping decisions deviate from the normative benchmark.

\section{Method}

Sample. Participants were 131 members of a university administered panel of consumers residing in the United States. They completed the study from their home, using personal computers connected to the Internet, and they did so in exchange for a guaranteed compensation of $\$ 3$ plus an additional amount of up to $\$ 5$ (see below). The average age of participants was 39.7 years, $69.4 \%$ of them were female, and $62.2 \%$ held a bachelors (or higher) academic degree.

Procedure and Stimuli. The experiment involved a sequential search task (with recall), where alternatives of known utility-i.e., monetary value-were revealed one at a time. Participants were told that they were free to inspect as many alternatives as they wished, instructed to choose one of the alternatives revealed in the course of their search, and informed that they would receive the monetary value of their chosen alternative as an additional payment upon completion of the experiment. Prior to starting the search task, participants were provided with detailed instructions (including a description of the monetary incentive), and they were required to pass a test of understanding of these instructions consisting of four multiple-choice questions.

Each alternative was characterized by a horizontal line displayed on the computer screen. The length of the line represented the alternative's monetary value. The values (line lengths) ranged from $\$ 0.01$ to $\$ 5.00$ (1 to 500 pixels). At each search stage, and separately for each participant, the value of the current alternative was determined by an independent random draw from a uniform distribution. Participants had complete and accurate information about the process used to generate these values. They incurred a monetary search cost for each inspected alternative. Each participant was randomly assigned to one of two treatment conditions for the entire experiment-a search cost of either $\$ 0.01$ or $\$ 0.05$ per alternative.

At each stage of the search, participants indicated (1) whether the current alternative was the best one they had seen up to that point and (2) whether they wished to inspect another alternative or stop searching and select (the one most recently identified as) the best alternative they had seen as their final choice. Upon completion of the experiment, each participant received the amount of money associated with the alternative he or she had selected minus the total cost of search he or she had incurred.

Dependent Variables. The set of dependent variables in the product comparison and stopping models parallel those used in Experiment 1. At each stage of the search process, we observed whether a participant marked the current alternative as the best one among those inspected up to that point, and this served as the dependent variable in the product comparison model. That is, we obtained a measure of whether a participant preferred the newly inspected alternative to the best previously encountered one. In addition, we observed at each stage whether a participant chose to terminate the search, and this was used as the dependent variable in the estimation of the stopping model.

Predictor Variables in the Product Comparison Model. The set of variables that was used to model both the normative and the proposed behavioral influences in the product comparison model was largely the same as in Experiment 1. The key difference is that the utility of each alternative and the (monetary) cost of search were known in Experiment 2. Consequently, we were able to use the objective values of 
the inspected alternatives (instead of estimated product utilities) and to directly calculate the normative reservation values.

Predictor Variables in the Stopping Model. In Experiment 2, the normative policy was for a participant to continue searching until a value greater than or equal to the reservation value was observed. Because the reservation value varied only as a function of the (manipulated) cost of search, model estimation was simplified as the reservation value was known for each participant. We still allowed for systematic differences in search cost across individuals with an individual-specific random effect. This effect was included additively in the model. The proposed behavioral influences (i.e., focalism and local contrast) were captured using variables that are analogous to those used in Experiment 1.

Model Estimation. because of the highly controlled nature of Experiment 2 (with alternatives of known value drawn from a known distribution and monetary search cost), the normative model componentsi.e., $U_{t i}, U_{t i}^{\max }$, and $r_{t i}$-are known exactly. For utilities known to be uniformly distributed between 0 and 500 (cents), the reservation value, as prescribed by the normative model, is $500-\sqrt{1,000 \cdot c_{i}}$ and, thus, 468 or 429 when the cost of search is 1 or 5 cent(s), respectively. ${ }^{12}$ Normatively, participants should terminate the search as soon as they inspect a value that exceeds the reservation value. The stopping and product comparison models were again estimated simultaneously. However, unlike the model for Experiment 1, the one estimated for Experiment 2 does not need to account for consumer learning about the distribution of product utilities over the course of the search.

\section{Results}

Descriptive Statistics. On average, participants inspected the descriptions of 5.9 alternatives. Of the 131 participants, $64.1 \%$ stopped too early in that they terminated their search before encountering an alternative that exceeded their reservation value, $26.7 \%$ searched in accordance with the normative model and stopped once they observed an alternative that exceeded their reservation value, and the remaining $9.2 \%$ continued their search even after inspecting an alternative that exceeded their reservation value. The mean number of alternatives searched by those who stopped too early, in accordance with the normative model, and too late are 4.3, 7.5, and 12.9, respectively. The finding that, overall, participants did not

\footnotetext{
${ }^{12}$ This equation for the reservation utility follows from the (more general) case with learning by letting the weight $W$ of the prior go to infinity, resulting in $(W+t) / W=1)$, and substituting the appropriate range for the uniform distribution.
}

search enough holds for both levels of search cost-in each of the two conditions, the number of alternatives inspected was about half the optimal number implied by the normative model for that level of search cost.

The average value of the best alternative that participants encountered in their search was $\$ 4.27$ and that of the product they chose was $\$ 4.23$. Taking into account the cost of search, we can also determine the expected payoff obtained by an individual who behaves according to the normative model, which is $\$ 4.49$. The actual average payoff earned by participants in the experiment was $\$ 4.06$.

Product Comparison Decision. The results for the product comparison model are summarized in the top part of Table 2. First, the normative model suggests that the decision as to whether the currently inspected alternative is more attractive than the best previously encountered one should be based only on the difference in utility between these two alternatives. This effect is supported by our data $(\beta=26.95, t=4.26)$.

The first of our proposed behavioral influences on the product comparison decision in sequential search is that consumers are unduly influenced by the attractiveness of the currently inspected alternative. The evidence obtained in Experiment 2 provides support for such a focalism effect in product comparison. Above and beyond the normative effect of the difference in utility between the currently inspected and the best previously encountered alternative, the utility of the current alternative has a significant positive impact on the probability of that alternative being adopted as the most attractive one encountered thus far $(\beta=11.76, t=3.32)$.

The other candidate behavioral influence is that the product comparison decision is influenced by the

Table 2 Model Estimates (Experiment 2)

\begin{tabular}{lrr}
\hline Predictor variable & $\beta$ & $t$-value \\
\hline Product comparison model & $-7.12^{*}$ & 3.17 \\
$\quad$ Intercept & $26.95^{*}$ & 4.26 \\
N Difference between current value and & & \\
$\quad$ highest value seen previously & $11.76^{*}$ & 3.32 \\
B Focalism (differential impact of current alternative) & 3.80 & 0.41 \\
B Local contrast: Improvement vs. prior alternative & -0.38 & -0.28 \\
B Local contrast: Deterioration vs. prior alternative & $3.51^{*}$ & 3.16 \\
$\quad \sigma\left(v^{P C}\right)$ & & \\
Stopping model & $-2.03^{*}$ & -5.93 \\
$\quad$ Intercept & 0.97 & 0.63 \\
$\quad$ Correction for utility normalization & $4.24^{*}$ & 2.53 \\
N Difference between highest value seen & & \\
$\quad$ and reservation value & 1.65 & 0.88 \\
B Focalism (differential impact of current alternative) & $1.40^{*}$ & 2.26 \\
B Local contrast: Improvement vs. prior alternative & $0.71^{*}$ & 3.17 \\
$\quad \sigma\left(v^{S}\right)$ & & \\
\hline
\end{tabular}

Note. N: normative influence; B: behavioral influence.

*Parameter estimate significant at $p<0.05$. 
difference in utility between the currently inspected alternative and the one seen just prior to it even when the latter is not the best among the previously observed alternatives and is, thus, normatively irrelevant. Such local contrasts are not significant in the product comparison model, both when they represent an improvement and when they constitute a deterioration relative to the preceding alternative.

Stopping Decision. The results for the stopping model are also summarized in Table 2. First, the normative effect is supported-the probability of terminating the search at a given stage increases as the difference between the highest value that an individual has observed in the course of the search and the reservation value increases $(\beta=4.24, t=2.53)$.

The first of the two proposed behavioral influences on the stopping decision is that the attractiveness of the current alternative is overweighed. In particular, we have predicted that the utility of the best among all observed alternatives has a stronger (positive) effect on consumers' probability of terminating the search at a given stage when that alternative happens to be the currently inspected one. Such a focalism effect on stopping is not supported by our data.

Moreover, we have proposed that the stopping decision in sequential product search is influenced by the local improvement in utility in the currently inspected alternative relative to the one seen just prior to it, which is normatively irrelevant. This local contrast effect is significant $(\beta=1.40, t=2.26)$, suggesting that, all else being equal, observing an alternative that is superior to the one they saw just before it renders individuals more likely to terminate their search.

Comparative Model Fit. As an overall test of the behavioral influences, we also estimated the baseline normative model as a benchmark. This model was identical to the full behavioral model with the exception that it did not include the proposed set of behavioral effects. The behavioral model fits the data significantly better than the baseline normative model-both based on a likelihood-ratio test $\left(\chi^{2}=145.0, d f=6, p<0.0001\right)$ and in terms of BIC (655.8 for the behavioral model versus 771.5 for the normative model), indicating that the behavioral effects contribute substantially to explaining the product comparison and stopping decisions. To test for heterogeneity in the behavioral effects across individuals, we also estimated a latent-class model with class-specific coefficients for both the normative and the behavioral influences. Such a model with two latent classes is inferior (BIC of 678.0) to the homogeneous behavioral model (BIC of 655.8) for these data. As an absolute measure of fit, McFadden's $R$-squared for the behavioral model is 0.59 , which is substantially higher than in Experiment 1. This difference reflects the fact that Experiment 1 was designed to examine the proposed behavioral influences in a naturalistic setting that entailed considerably more unobservable factors.

\section{Discussion}

The results of Experiment 2 provide further support for the proposed behavioral influences. As in Experiment 1 , the focalism effect is significant in the product comparison model, whereas the local contrast effect is again significant in the stopping model. Thus, we find strong convergence between the results of the two studies.

We illustrate the magnitude of the key behavioral influences observed in Experiment 2 using the same approach as in Experiment 1. That is, for a given behavioral effect, we again examine how variation in the variable that captures it affects searchers' micro decisions ceteris paribus (i.e., with all other variables in the model held constant), and we do so based on the estimated parameters of the product comparison and stopping models reported in Table 2 .

To provide a sense of the magnitude of the focalism effect on product comparison decisions, we examine the probability of the current alternative being selected as the most preferred one thus far for both the 10th and the 90th percentile value of this alternative's value when the latter is equal to the value of the best previously encountered alternative. Normatively, when the current alternative and the best prior alternative are equally attractive, the inclination to prefer the current one should not be affected by their absolute attractiveness. However, the resulting probabilities are in fact vastly different-they are $6.0 \%$ and $82.4 \%$ for the 10 th and the 90th percentile values, respectively. Thus, our findings indicate a much greater sensitivity to the utility of the current alternative than to that of the best prior one. To further quantify this behavioral influence, our model estimates imply that, if the best previously encountered alternative was at the median of the utility distribution, the currently inspected one would only have to be at the 6th percentile to be equally likely to be selected as the most preferred alternative thus far.

To illustrate the local contrast effect on stopping decisions, we examine the probability of terminating the search at the current stage for the 10th and the 90th percentiles of the value of the alternative inspected just prior to the current one, while holding both the value of the current alternative and that of the best one encountered thus far constant at the 90th percentile. The resulting stopping probabilities are $70.7 \%$ and $54.3 \%$ for the 10 th and the 90th percentiles of the previously inspected alternative's value, respectively. This provides a sense of the magnitude of the local contrast effect because, normatively, the attractiveness of the alternative encountered just prior to 
the current one should not affect a searcher's stopping decision. To further quantify this effect, if the previous alternative was at the 10th percentile of the utility distribution, the current alternative would only have to be at the 42nd percentile to achieve the same stopping probability as when the utilities of both the current and the previous alternative are at the median. Thus, all else being equal, a local increase in attractiveness from one inspected alternative to the next in the search sequence unduly enhances the inclination to terminate the search.

Finally, as in Experiment 1, the normative effects are again significant both in the product comparison model and in the stopping model along with the behavioral influences, which further corroborates the conclusion that the latter are an important extension of the normative theory of sequential search and not a substitute for it.

\section{General Discussion}

We have introduced and tested a behavioral model of consumer product search that extends a baseline normative model of sequential search by incorporating a class of nonnormative influences that are local in the sense that they reflect consumers' disproportionate sensitivity to, or "zooming in" on, recently encountered alternatives. Converging evidence obtained via different experimental paradigms-one involving consumer search for a multiattribute product in a setting of high external validity (Experiment 1) and the other using a much more tightly controlled procedure that ensures high internal validity (Experiment 2)-provides strong support for the proposed behavioral influences, along with effects implied by the normative theory. Our findings thus confirm the key proposition that consumer product search behavior is jointly governed by normative principles and (local) nonnormative influences.

The overall pattern of results is consistent across the two experiments. First, in addition to the normative effect, product comparison decisions in search reflect a focalism effect such that consumers respond excessively to the attractiveness of the currently inspected product, at the expense of all other alternatives. Second, along with normative considerations, stopping decisions are affected by local contrast effects whereby consumers are unduly influenced by the difference in attractiveness between the current product and the one encountered just prior to it. This differential susceptibility of the product comparison and stopping decisions to the two local behavioral influences may reflect a difference in focus between these micro decisions in consumer product search. Exploring this difference in greater depth would be a promising avenue for future work.
The present work reveals a substantial behavioral path dependence in search-based consumer choices in the form of local influences. That is, the particular order in which consumers encounter candidate products-whether this order occurs naturally or is deliberately designed by an interested party-has a systematic impact on their product comparison and stopping decisions in sequential search. For instance, a strong contrast in the attractiveness of two contiguously encountered products experienced early on in the search process can result in the premature termination of the search (relative to the normative policy), whereas the persistent absence of such an experience can lead to excessive search. In addition, our findings suggest the possibility of a systematic consumer preference reversal between two products merely as a result of the order in which they are inspected and what other products they are embedded among within a search sequence.

More generally, the present research heeds the call to develop behavioral models that shed light on nonnormative decision rules that consumers use in dynamic contexts where, as in product search, they are required to make sequences of interdependent decisions (Adamowicz et al. 2008). We have done so by using an integrative approach and developing a model of consumer product search that augments a baseline normative model with behavioral influences that reflect searchers' undue sensitivity to recently encountered (i.e., local) alternatives.

The findings reported in this article have important implications for sellers. For instance, when consumers engage in a sequential search of several attractive products, the one they are inspecting at the moment enjoys a distinct advantage in the sense that it is more likely to be preferred merely as a result of being the focal product. As this advantage vanishes as soon as the consumer moves on to inspecting the next product, a seller should seek to capitalize on this momentary opportunity and close the deal while its product is still the focal one. Another implication of our findings is that, to the extent that a vendor's goal is to prevent (or at least reduce) further search once consumers have inspected its own product, it should seek to influence the nature of the competing alternative that consumers encounter immediately prior to inspecting its offering-for example by ensuring that consumers are exposed to a distinctly unattractive alternative just before they see the firm's product.

In terms of implications for consumers, our findings suggest that individuals' search behavior is quite susceptible to nonnormative forces. The identification of important sources of such, presumably unwanted, influence on purchase decisions should help consumers counteract these nonnormative inclinations and improve the quality of their search-based 
product choices. Given their natural tendency to be unduly influenced by recently inspected alternatives when deciding which is their most preferred product and whether to stop searching at a given stage, consumers engaging in sequential product search would be well advised to make a strong effort to accurately remember-or even record-which of the previously inspected products is the most attractive one to them, and to not allow their decisions to be influenced by recently encountered unattractive alternatives.

Some limitations of the present research are worth noting, along with opportunities for future research. First, although we used different experimental paradigms in an effort to attain both high internal validity and high external validity, our findings may not fully generalize to search decisions in the wild. For instance, the stakes tend to be higher in real-world decisions, and this might result in greater motivation and more search effort than we observed in our experiments. Therefore, it would be worthwhile to further test our behavioral model of consumer product search using field data (e.g., observed search behavior at an Internet shopping site or survey data on consumers' product search activities). Another promising avenue for further work would be to extend our model to situations where consumers have partial information about specific alternatives prior to their inspection.

It would also be interesting to examine whether consumers tend to update their preferences as they engage in sequential product search. A common phenomenon in consumer choice behavior is that individuals tend to construct their preferences in response to the specific properties of the choice tasks they face (see Bettman et al. 1998). Similarly, consumers might also construct their preferences during sequential product search. Thus, enhancing our understanding of the connection between product search and preference formation is an important objective for future research.

Another important area for future research is the impact of decision support technologies on consumer product search behavior. For example, one potentially powerful means of assisting consumers with the type of complex search tasks examined in the present research is to provide them with decision aids that facilitate comparisons of the currently observed product with the best one encountered up to that point. Although it has been shown that such product comparison tools can significantly improve consumer decision making (Häubl and Trifts 2000), it would also be interesting to examine whether and how these decision support technologies moderate the behavioral influences on consumer search behavior introduced here.

Finally, because the specific properties of the search environment-what is known about the distribution of utilities, whether the search is conducted with or without recall, etc.-influence what constitutes normatively optimal search behavior (Adam 2001), it would be worthwhile to replicate the behavioral effects reported here in other types of search environments. Moreover, although it is likely that there are instances where optimal search strategies do not have a reservation utility property (see Albright 1977), the question of how consumers search under such circumstances has not been addressed to date. We hope that the present work provides an impetus for the rigorous investigation of consumer search behavior in such contexts.

\section{Acknowledgments}

The authors gratefully acknowledge the support of the Canada Research Chairs program, the R. K. Banister Professorship in Electronic Commerce, the Social Sciences and Humanities Research Council of Canada, the Killam Research Fund, and the University of Alberta School of Retailing to the first author, and of the Netherlands Organization for Scientific Research to the second author. Valuable assistance with the data collection for Experiment 1 was provided by CentERdata and by a grant from the Faculty of Economics and Business Administration at Tilburg University. The authors thank the editor, the associate editor, and the team of reviewers, as well as Ulf Böckenholt, Eric Johnson, Keri Kettle, Monica Popa, Bob Price, Brian Ratchford, and Rami Zwick for their thoughtful comments and suggestions.

\section{Appendix. Baseline Normative Model}

In line with Rothschild (1974) and Bikhchandani and Sharma (1996), we assume that consumers update their beliefs about the distribution of product utilities in the market using Bayesian updating. We assume a continuous distribution, but for expositional clarity first derive the updating rule for a discrete distribution. This then naturally translates to the case of a continuous distribution (cf. Bikhchandani and Sharma 1996).

Let the overall utilities that a consumer ${ }^{13}$ attaches to products in the market be denoted by $U_{1}, U_{2}, \ldots, U_{m}$, with $U_{j}<U_{j+1}$ for $j=1,2, \ldots, m-1$. The probability distribution over these utilities is a multinomial distribution with unknown parameters $\pi_{1}, \pi_{2}, \ldots, \pi_{m} \geq 0$ and $\sum_{j=1}^{m} \pi_{j}=1$. If the consumer holds a prior distribution on $\pi_{1}, \pi_{2}, \ldots, \pi_{m}$ that is Dirichlet with parameters $\delta$, then for $k=1, \ldots, m$ the prior distribution of the probability $\pi_{k}$ of observing a product with utility $U_{k}$ is multinomial with parameters $\delta_{k} / \sum_{j=1}^{m} \delta_{j}$. Because the Dirichlet is the conjugate prior of the multinomial distribution, the posterior distribution is also Dirichlet. In particular, after observing a utility $U_{l}$, the parameters of the posterior distribution are updated to $\delta_{1}, \delta_{2}, \ldots, \delta_{l}+1, \ldots, \delta_{m}$. Suppose that a consumer has inspected $t$ products and the highest observed utility is $U_{l}$,

${ }^{13}$ For ease of exposition, the subscript $i$ (denoting the consumer) is omitted in this appendix. 
then the updated probabilities $\pi_{k}, k=l+1, \ldots, m$, of finding a product with a higher utility equal $\left.\delta_{k} / \sum_{j=1}^{m} \delta_{j}+t\right)$. Hence, the reservation utility cannot increase with search.

Bikhchandani and Sharma (1996) extended this model to the Dirichlet process, which is a generalization of the Dirichlet distribution to a stochastic process with an infinite set of utilities (Ferguson 1973, 1974). In particular, assuming a uniform prior on $[0,1]$ with parameter $W$ reflecting the weight attached to the initial prior, after inspecting $t$ products, the updated probability density function for products of utility exceeding the highest utility observed thus far $\left(U_{t}^{\max }\right)$ is then given by

$$
\begin{aligned}
f_{t}(U) & =I_{\left[0, U_{t}^{\max }\right]}(U) \cdot W /\left(\int_{0}^{1} W d x+t\right) \\
& =I_{\left[0, U_{t}^{\max }\right]}(U) \cdot W /(W+t),
\end{aligned}
$$

based on Bikhchandani and Sharma (1996, p. 358). This is the updated distribution function consumers use at stage $t$ to assess the expected gains of continuing the search.

Calculation of the expected gain of continuing the search involves two components-(1) the expected gain conditional on finding a more attractive product and (2) the probability of finding a more attractive product.

The expected gain in utility, assuming a more attractive product is found, is the expected utility of that product minus the utility of the most attractive one observed thus far. As we have a uniform distribution of product utilities for the range $\left[U_{t}^{\max }, 1\right]$, the expected utility of a more attractive product is $\left(1+U_{t}^{\max }\right) / 2$, and hence the expected gain equals $\left(1-U_{t}^{\max }\right) / 2$. The probability of finding a more attractive product, based on the updated density function given above, is

$$
\int_{U_{t}^{\max }}^{1} f_{t}(U) d U=\left(1-U_{t}^{\max }\right) \cdot W /(W+t) .
$$

The expected gain of continuing the search is the product of the probability of finding a more attractive product and the expected gain conditional on finding such a product. Combining the results above yields

$$
U^{\mathrm{CONT}}=1 / 2 \cdot\left(1-U_{t}^{\max }\right)^{2} \cdot W /(W+t)
$$

The reservation utility $r_{t}$ at stage $t$ is that value of $U_{t}^{\max }$ that results in a value of $U_{t}^{\mathrm{CONT}}$ equal to the cost of search $c$, and $r_{t}$ is thus defined by

$$
c=1 / 2 \cdot\left(1-r_{t}\right)^{2} \cdot W /(W+t) .
$$

One obtains $\left(r_{t}=1-\sqrt{2(W+t) / W} \cdot \sqrt{c}\right)$ through transformation, which is used in model estimation.

\section{References}

Adam, K. 2001. Learning while searching for the best alternative. J. Econom. Theory 101(1) 252-281.

Adamowicz, W., D. Bunch, T. A. Cameron, B. G. C. Dellaert, M. Hanneman, M. Keane, J. Louviere, R. Meyer, T. Steenburgh, J. Swait. 2008. Behavioral frontiers in choice modeling. Marketing Lett. 19(3-4) 215-228.

Albright, S. C. 1977. A Bayesian approach to a generalized house selling problem. Management Sci. 24(4) 432-440.
Arend, L. E., B. Spehar. 1993. Lightness, brightness, and brightness contrast: 1. Illuminance variation. Perception Psychophysics 54(4) 446-456.

Bearden, J. N., R. O. Murphy, A. Rapoport. 2005. A multi-attribute extension of the secretary problem: Theory and experiments. J. Math. Psych. 49(5) 410-422.

Ben-Akiva, M., D. McFadden, T. Gärling, D. Gopinath, J. Walker, D. Bolduc, A. Börsch-Supan et al. 1999. Extended framework for modeling choice behavior. Marketing Lett. 10(3) 187-203.

Bettman, J. R., M. F. Luce, J. W. Payne. 1998. Constructive consumer choice processes. J. Consumer Res. 25(3) 187-217.

Bikhchandani, S., S. Sharma. 1996. Optimal search with learning. J. Econom. Dynam. Control 20(1-3) 333-359.

Brannon, J. I., M. F. Gorman. 2002. The effects of information costs on search and convergence in experimental markets. J. Econom. Behav. Organ. 47(4) 375-390.

Buehler, R., D. Griffin, M. Ross. 1994. Exploring the "planning fallacy": Why people underestimate their task completion times. J. Personality Soc. Psych. 67(3) 366-381.

Cox, J. C., R. L. Oaxaca. 1989. Laboratory experiments with a finitehorizon job-search model. J. Risk Uncertainty 2(3) 301-329.

Elrod, T., J. J. Louviere, K. S. Davey. 1992. An empirical comparison of ratings-based and choice-based conjoint models. J. Marketing Res. 29(August) 368-377.

Erdem, T., M. P. Keane, T. S. Öncü, J. Strebel. 2005. Learning about computers: An analysis of information search and technology choice. Quant. Marketing Econom. 3(3) 207-247.

Ferguson, T. S. 1973. A Bayesian analysis of some nonparametric problems. Ann. Statist. 1(2) 209-230.

Ferguson, T. S. 1974. Prior distributions on spaces of probability measures. Ann. Statist. 2(4) 615-629.

Gilbert, D. T., T. D. Wilson. 2007. Prospection: Experiencing the future. Science 317(5843) 1351-1354.

Harrison, G. W., P. Morgan. 1990. Search intensity in experiments. Econom. J. 100(401) 478-486.

Häubl, G., V. Trifts. 2000. Consumer decision making in online shopping environments: The effects of interactive decision aids. Marketing Sci. 19(1) 4-21.

Hauser, J. R., G. L. Urban, B. D. Weinberg. 1993. How consumers allocate their time when searching for information. J. Marketing Res. 30(4) 452-466.

Hey, J. D. 1982. Search for rules for search. J. Econom. Behav. Organ. 3(1) 65-81.

Hey, J. D. 1987. Still searching. J. Econom. Behav. Organ. 8(1) 137-144.

Heyman, J., B. A. Mellers, S. Tishcenko, A. Schwartz. 2004. I was pleased a moment ago: How pleasure varies with background and foreground reference points. Motivation Emotion 28(1) 65-83.

Hogarth, R. M., H. J. Einhorn. 1992. Order effects in belief updating: The belief-adjustment model. Cognitive Psych. 24(1) 1-55.

Houser, D., J. Winter. 2004. How do behavioral assumptions affect structural inference? Evidence from a laboratory experiment. J. Business Econom. Statist. 22(1) 64-79.

Johar, G., K. Jedidi, J. Jacoby. 1997. A varying-parameter averaging model of online brand evaluations. J. Consumer Res. 24(2) 232-247.

Kahneman, D., D. T. Miller. 1986. Norm theory: Comparing reality to its alternatives. Psych. Rev. 93(2) 136-153.

Kogut, C. A. 1990. Consumer search behavior and sunk costs. J. Econom. Behav. Organ. 14(3) 381-392.

Kruger, J., M. Evans. 2004. If you don't want to be late, enumerate: Unpacking reduces the planning fallacy. J. Experiment. Soc. Psych. 40(5) 586-598.

McFadden, D. 1986. The choice theory approach to market research. Marketing Sci. 5(4) 275-297. 
Mellers, B. A. 2000. Choice and the relative pleasure of consequences. Psych. Bull. 126(6) 910-924.

Meyer, R. J. 1982. A descriptive model of consumer information search behavior. Marketing Sci. 1(1) 93-121.

Moon, P., A. Martin. 1990. Better heuristics for economic search: Experimental and simulation evidence. J. Behav. Decision Making 3(3) 175-193.

Moon, P., A. Martin. 1996. The search for consistency in economic search. J. Econom. Behav. Organ. 29(2) 311-321.

Morris, C. N. 1983. Parametric empirical Bayes inference: Theory and applications. J. Amer. Statist. Assoc. 78(381) 47-55.

Pessoa, L., E. Mingolla, H. Neumann. 1995. A contrast- and luminance-driven multiscale network model of brightness perception. Vision Res. 35(15) 2201-2223.

Phipps, A. G., R. J. Meyer. 1985. Normative versus heuristic models of residential search behavior: An empirical comparison. Environment Planning A 17(6) 761-776.

Putrevu, S., B. T. Ratchford. 1997. A model of search behavior with an application to grocery shopping. J. Retailing 73(4) 463-487.

Ratchford, B. T., N. Srinivasan. 1993. An empirical investigation of returns to search. Marketing Sci. 12(1) 73-87.

Reid, R. C., R. Shapley. 1988. Brightness induction by local contrast and the spatial dependence of assimilation. Vision Res. 28(1) 115-132.

Rosenfield, D. B., R. D. Shapiro, D. A. Butler. 1983. Optimal strategies for selling an asset. Management Sci. 29(9) 1051-1061.

Rothschild, M. 1974. Searching for the lowest price when the distribution of prices is unknown. J. Political Econom. 82(4) 689-711.
Schkade, D. A., D. Kahneman. 1998. Does living in California make people happy? A focusing illusion in judgments of life satisfaction. Psych. Sci. 9(5) 340-346.

Schotter, A., Y. M. Braunstein. 1981. Economic search: An experimental study. Econom. Inquiry 19(1) 1-25.

Schwarz, N. 1990. Feelings as information: Informational and motivational functions of affective states. R. Sorrentino, E. T. Higgins, eds. Handbook of Motivation and Cognition, Vol. 2. Guilford Press, New York, 527-561.

Shu, S. B. 2008. Future-biased search: The quest for the ideal. J. Behav. Decision Making 21(4) 352-377.

Sonnemans, J. 1998. Strategies of search. J. Econom. Behav. Organ. 35(3) 309-332.

Stern, S. 1997. Simulation-based estimation. J. Econom. Lit. 35(4) 2006-2039.

Stigler, G. J. 1961. The economics of information. J. Political Econom. 69(3) 213-225.

Suh, E., E. Diener, F. Fujita. 1996. Events and subjective wellbeing: Only recent events matter. J. Personality Soc. Psych. 70(5) 1091-1102.

Train, K. 2003. Discrete Choice Methods with Simulation. Cambridge University Press, New York.

Wilson, T. D., D. T. Gilbert. 2005. Affective forecasting: Knowing what to want. Current Directions Psych. Sci. 14(3) 131-134.

Wilson, T. D., T. Wheatley, J. M. Meyers, D. T. Gilbert, D. Axsom. 2000. Focalism: A source of durability bias in affective forecasting. J. Personality Soc. Psych. 78(5) 821-836.

Zwick, R., A. Rapoport, A. K. C. Lo, A. V. Muthukrishnan. 2003. Consumer sequential search: Not enough or too much? Marketing Sci. 22(4) 503-519. 